\title{
Pharmacological agents with inherent anti-autophagic activity improve the cytotoxicity of imatinib
}

\author{
LISA C. CROWLEY, TRACEY R. O'DONOVAN, MICHELLE J. NYHAN and SHARON L. McKENNA
}

Leslie C. Quick Laboratory, Cork Cancer Research Centre, BioSciences Institute, University College Cork, Cork, Ireland

Received January 28, 2013; Accepted March 11, 2013

DOI: $10.3892 /$ or.2013.2377

\begin{abstract}
Resistance to tyrosine kinase inhibitors (TKIs) remains a limitation to the treatment of chronic myeloid leukaemia (CML), due in part, to the induction of autophagy. We examined whether disruption of autophagy with the pharmacological agents, brefeldin A, vincristine and chloroquine, improves the cytotoxicity of imatinib. In K562 CML cells, all drugs tested, in combination with imatinib impaired the expression or cellular distribution of LC3 and Beclin 1 (autophagy markers) and reduced the recovery of cells following drug withdrawal. The combination of imatinib and an agent that impedes autophagy demonstrates impressive potential as a more curative regime for CML.
\end{abstract}

\section{Introduction}

The Philadelphia chromosome, a hallmark of chronic myeloid leukaemia (CML), generates the fusion oncoprotein, Bcr-Abl, and a constitutively active tyrosine kinase $(1,2)$. Chronic phase CML is well managed in most patients with tyrosine kinase inhibitors (TKIs), most notably imatinib, whereas the advanced stage of CML known as blast crisis is a highly aggressive drug-resistant disease and is analogous to acute leukaemia (3).

Despite the success of imatinib for the treatment of CML, treatment failures due to resistance and relapse are still common. Bcr-Abl amplification, mutations or the acquisition of Bcr-Abl-independent mechanisms have been shown to play a role in the development of resistance (4). In addition, relapse is common when imatinib is withdrawn, even in patients who achieve a complete molecular response and this can re-emerge as the same imatinib susceptible disease (5). This highlights the need for potentially life-long treatment and the possibility of resistant disease emerging later on.

We and others have shown that the induction of autophagy in response to treatment with imatinib is associated with persistence and recovery of a subpopulation of leukaemia cells $(6,7)$. Autophagy is a survival mechanism initiated in

Correspondence to: Dr Sharon L. McKenna, Leslie C. Quick Laboratory, Cork Cancer Research Centre, BioSciences Institute, University College Cork, Cork, Ireland

E-mail: s.mckenna@ucc.ie

Key words: CML, Bcr-Abl, imatinib, autophagy response to cellular stress or starvation. It removes damaged organelles and aggregated proteins and can recycle cytoplasmic content (8). Inhibition of autophagy can significantly reduce the recovery of CML cells following imatinib treatment (7). This is one of the most promising strategies for improving the curative ability of imatinib.

Currently, there are no specific autophagy inhibitors. There are, however, a limited number of pharmacological agents that have been reported to disrupt processes or organelles required for effective autophagy. Combining such agents with imatinib may therefore compromise the autophagic recovery employed by leukaemic cells, potentially improving treatment regimens for CML. We evaluated three agents in combination with imatinib: chloroquine, vincristine and brefeldin A. Chloroquine, a lysosomotropic agent, damages lysosomal integrity leading to the inhibition of autophagosome turnover (9) and is currently in clinical trials in combination with imatinib for treatment of CML (10). The second agent in the present study, vincristine, is a microtubulin de-polymerising agent which arrests cells in the $\mathrm{G}_{2} / \mathrm{M}$ phase. Evidence suggests that cells undergoing mitosis are resistant to autophagic induction (11) and therefore, $\mathrm{G}_{2} / \mathrm{M}$ inhibitors may have anti-autophagic activity in combination regimes. Vincristine has also been reported to impede autophagy by disrupting the microtubulin network and inhibiting the trafficking required for autophagosome and lysosomal fusion (12). The third agent in this study, brefeldin A disrupts protein transport in the Golgi compartment (13). While this may lead to endoplasmic reticulum (ER) stress and potentially increase autophagy, brefeldin A has been reported to block an alternative autophagic pathway (14). This may be due to involvement of the Golgi region in the initiation of certain types of autophagy.

In the present study, we investigated the autophagic response of K562 CML cells following imatinib treatment alone and combination treatment with potential autophagy-disrupting agents. Our data indicate that the early implementation of imatinib combined with autophagy-disrupting agents, reduces cell recovery and may potentially improve the curative ability of imatinib in CML patients.

\section{Materials and methods}

Cell culture/reagents. K562 cells (blast crisis CML) were obtained from Deutsche Sammlung von Mikroorganismen 
und Zellkulturen GmbH (DSMZ) and were maintained in RPMI-1640 medium, HEPES modification, supplemented with $10 \%$ foetal calf serum, $1 \%$ penicillin and streptomycin (Sigma-Aldrich, Ireland Ltd., Dublin, Ireland) and $2 \mathrm{mmol} / 1$ L-glutamine (Gibco-BRL, Paisley, UK) cultured at $37^{\circ} \mathrm{C}$ in $5 \% \mathrm{CO}_{2}$. Chloroquine, vincristine and brefeldin A were all from Sigma-Aldrich Ireland Ltd. Imatinib was obtained from Novartis Pharma AG (Basel, Switzerland).

Cell viability and cell cycle analysis. Cells were resuspended in PBS with $50 \mu \mathrm{g} / \mu 1$ propidium iodide (PI; Sigma-Aldrich Ireland). FL-2 fluorescence was measured using a FACSCalibur ${ }^{\circledR}$ flow cytometer (Becton-Dickinson, Franklin Lakes, NJ, USA). Statistical significance was determined using the Student's t-test. P-values of $<0.05$ were considered statistically significant. For the prolonged viablity analysis, following the initial 24-h treatment, the drug was removed and cells were resuspended in fresh media. Viability was assessed over the subsequent 5 days. For cell cycle analysis, cells were fixed in $70 \%$ ethanol prior to PI staining.

Cell morphology and immunofluorescence. For cell morphology, cytospun cells were stained using Pro-Diff (Braidwood Laboratories, Ireland). For immunofluorescence staining, the cytospun cells were fixed in $4 \%$ paraformaldelyde for $20 \mathrm{~min}$ and washed in PBS. Permeabilisation and blocking were carried out using $0.005 \%$ Saponin/PBS and 0.2\% BSA/PBS, respectively. For antibody staining, LC3 (Abgent, San Diego, CA, USA) and Beclin 1 (Cell Signaling Technology, UK) were incubated (1:200) for $1 \mathrm{~h}$ at room temperature. After incubation with the appropriate secondary Alexa Fluor antibody, samples were mounted with Gold Plus with DAPI antifade reagent (Invitrogen, Dublin, Ireland). Images were captured using a DP70 digital microscope camera and Olympus DP-Soft823 version 3.2 software. All images are representative of at least three separate experiments.

\section{Results}

Distribution of LC3 and Beclin 1 following treatment with chloroquine, vincristine and brefeldin A with and without imatinib. We previously demonstrated that induction of autophagy in K562 CML cells limits the cytotoxic effect of imatinib and facilitates the recovery of treated cells (6). Viability and morphologic data for K562 cells are shown in Fig. 1A to enable comparison with the subsequent combination treatments. In the present study, we examined whether combinations of imatinib with potential autophagy-disrupting agents, chloroquine, vincristine and brefeldin A, alter expression of LC3 and Beclin 1 (autophagy markers).

Chloroquine. K562 cells were treated with $5 \mu \mathrm{M}$ imatinib, $25 \mu \mathrm{M}$ chloroquine or a combination of both for $24 \mathrm{~h}$. Cells treated with chloroquine alone displayed increased LC3 staining when compared to that in the control K562 cells (Fig. 1B-ii, upper panel) which was likely due to the inhibition of autophagosome turnover. K562 cells treated with chloroquine and imatinib together also showed increased LC3 expression in some cells when compared to the controls. This expression, however, was significantly reduced when compared to that in cells treated with either agent alone and was localised to a peri-nuclear region (Fig. 1B-ii, lower panel), suggesting impairment at either initiation or trafficking in addition to compromised turnover. Beclin 1 expression was also increased in cells treated with imatinib, exhibiting a more peri-nuclear localised staining pattern (Fig. 1C-i, lower panel). Cells treated with chloroquine alone or the combination with imatinib displayed diminished staining of Beclin 1, again suggesting that initiation of autophagy may be impaired in addition to turnover (Fig. 1C-ii). The distribution of both markers suggests that autophagy was impeded in the chloroquine-treated cells.

Vincristine. We investigated whether vincristine disrupts imatinib-induced autophagy. Cells were treated for $24 \mathrm{~h}$ with $10 \mathrm{nM}$ vincristine, $5 \mu \mathrm{M}$ imatinib or a combination of both. K562 cells treated with vincristine alone demonstrated a mixed LC3 staining pattern. Cells displaying mitotic disturbances [NB irregular DAPI-stained nuclei (white arrows)] did not express LC3. In contrast, cells with normal nuclei exhibited LC3 staining analogous to that in the control cells or was slightly elevated (Fig. 1B-iii, upper panel). A similar pattern was observed in the combination-treated cells (Fig. 1B-iii, lower panel). Beclin 1 was also absent or significantly reduced in the majority of vincristine-treated cells displaying nuclear mitotic abnormalities (Fig. 1C-iii, upper panel). Cells treated with the combination of imatinib and vincristine demonstrated reduced overall Beclin 1 expression when compared to expression in the cells treated with imatinib alone (Fig. 1C-iii, lower panel). This reduced/absent Beclin 1 staining, particularly in the $\mathrm{G}_{2} / \mathrm{M}$-arrested cells, indicated a reduced capacity to initiate autophagy.

Brefeldin A. K562 cells were treated with $1 \mu \mathrm{g} / \mathrm{ml}$ brefeldin A (BFA), $5 \mu \mathrm{M}$ imatinib or a combination of both for $24 \mathrm{~h}$. Cells treated with BFA alone showed reduced expression of LC3 when compared to that in the control cells (Fig. 1B-iv, upper panel). In cells treated with the combination of imatinib and BFA, LC3 staining was dramatically reduced compared to staining in cells treated with imatinib alone (Fig. 1B-iv, lower panel). Therefore, BFA impeded autophagy initiation in cells treated with imatinib. Notably, BFA treatment alone induced dramatic condensation of Beclin 1 to single perinuclear foci (Fig. 1C-iv, upper panel). Cell treated with the combination treatment exhibited a mixture of single discrete Beclin 1 foci and less condensed peri-nuclear localisation (Fig. 1C-iv, lower panel) suggesting that autophagy initiation was impaired.

Effect of chloroquine and imatinib treatment on cell viability and recovery. $\mathrm{K} 562$ cells were treated with $5 \mu \mathrm{M}$ imatinib, $25 \mu \mathrm{M}$ chloroquine or a combination of both for $24 \mathrm{~h}$ and cell viability was assessed over 5 days (Fig. 2A). K562 viability was unaffected by treatment with chloroquine alone at any time point. Forty-eight hours post treatment, the viability of K562 cultures treated with imatinib alone was $23.7 \pm 0.3 \%$. However, following the combination therapy, cell viability was much lower at $4.7 \pm 0.1 \%$, indicating a marked improvement when combining chloroquine and imatinib, which was not observed following the 24-h treatment. This combination effect was sustained as indicated by the absence of any recovery at day 5 (Fig. 2A). 

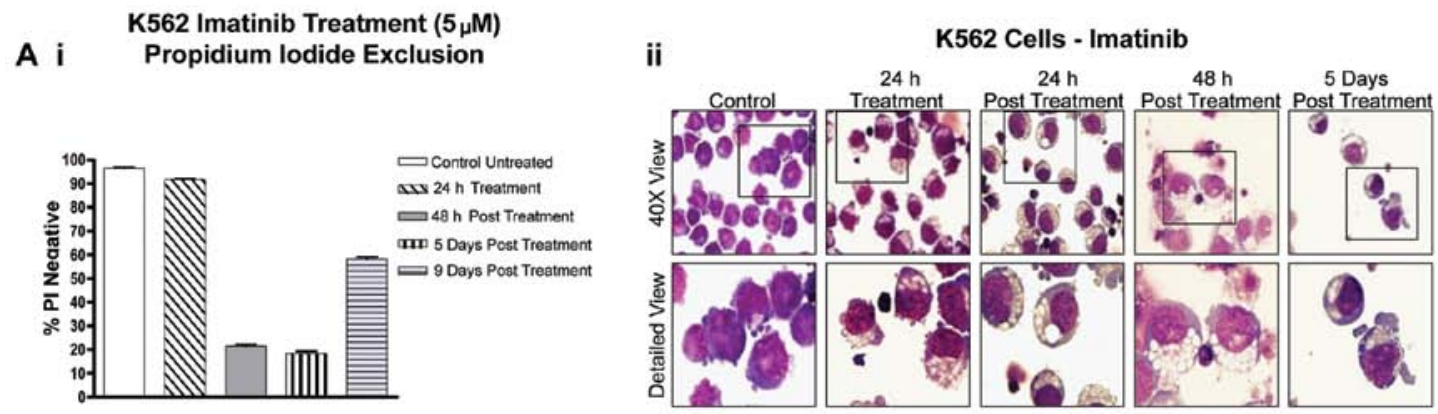

B

LC3
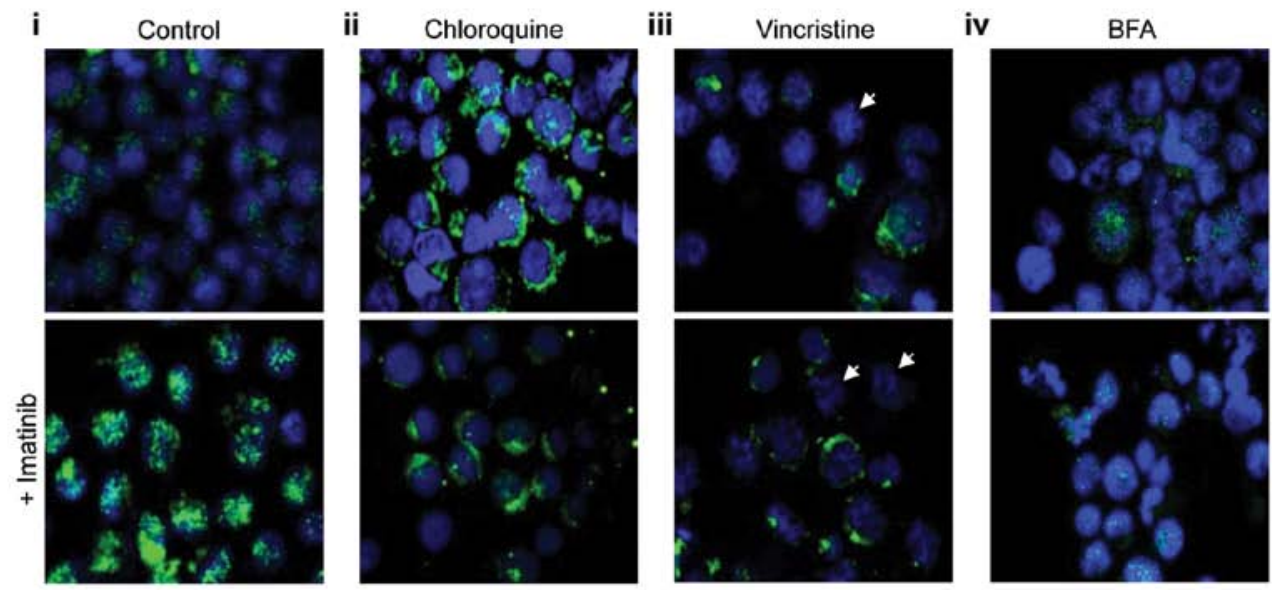

C

Beclin 1
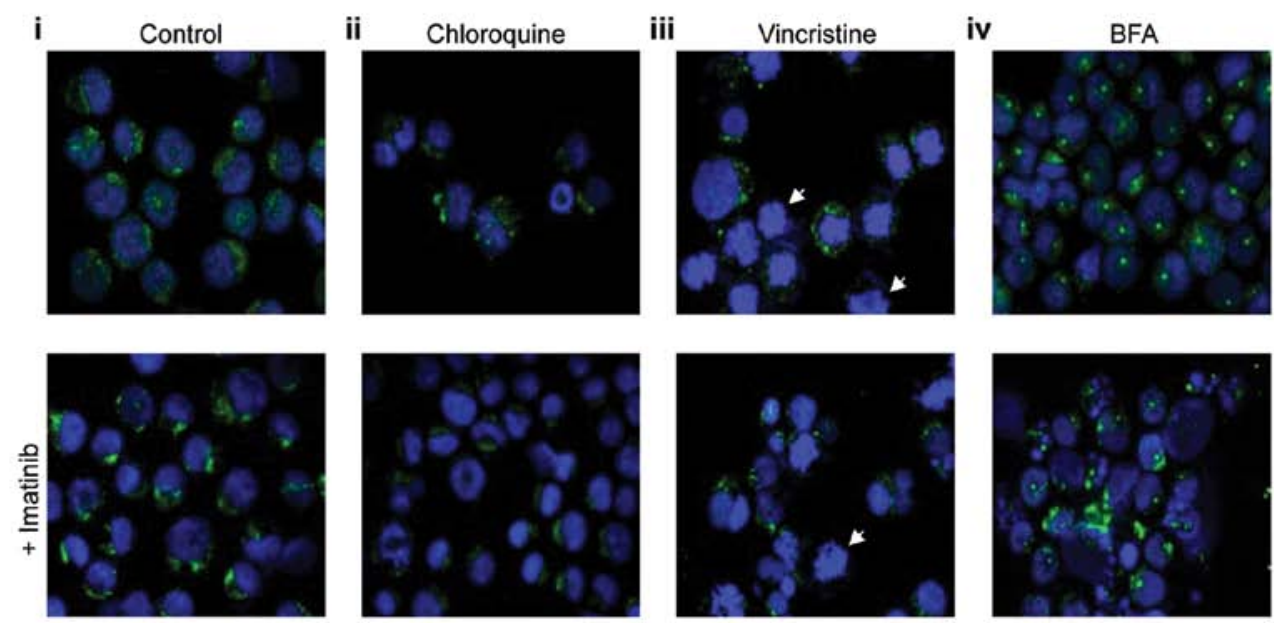

Figure 1. Effects of chloroquine, vincristine or brefeldin A alone or in combination with imatinib on LC3 and Beclin 1 expression in K562 cells. (A) Viability of K562 cells treated with $5 \mu \mathrm{M}$ imatinib for $24 \mathrm{~h}$ and up to 9 days following drug withdrawal. (i) Viability measured by PI exclusion. Bars indicate mean values \pm SE. (ii) Morphological analysis of control and treated cells. Prominent cytoplasmic vesicles are evident following a 24-h treatment with imatinib. Upper panels: magnification, x40; lower panels: enlarged section of upper panel indicated by a box. (B) Immunofluorescence analysis of LC3 staining of K562 cells. (i) Control cells. Cells treated with either (ii) $25 \mu \mathrm{M}$ chloroquine, (iii) $10 \mathrm{nM}$ vincristine or (iv) $1 \mu \mathrm{g} / \mathrm{ml}$ brefeldin A alone (upper panel) or in combination with $5 \mu \mathrm{M}$ imatinib (lower panels) for $24 \mathrm{~h}$. (C) Immunofluorescence analysis of Beclin 1 staining of K562 cells. (i) Control cells. Cells treated with either (ii) $25 \mu \mathrm{M}$ chloroquine, (iii) $10 \mathrm{nM}$ vincristine or (iv) $1 \mu \mathrm{g} / \mathrm{ml}$ brefeldin A alone (upper panels) or in combination with $5 \mu \mathrm{M}$ imatinib (lower panels) for $24 \mathrm{~h}$. In all treatments, the level or distribution of autophagy markers was impaired relative to the control and notably the elevation of LC3 by imatinib was substantially reduced when combined with any of the agents. White arrows, irregular DAPI-stained nuclei. BFA, brefeldin A.

Cells treated with chloroquine alone began to display vesicle accumulation in a small percentage of cells at $24 \mathrm{~h}$ (Fig. 2B-i, upper panel). At $48 \mathrm{~h}$ and 5 days post chloroquine treatment alone, cells showed an extensive build-up of vacuoles in their cytoplasm (Fig. 2B-i, middle and lower panel), consistent with previous reports (9). Notably, this did not significantly affect the viability of the culture.

Cells treated with the combination of imatinib and chloroquine for $24 \mathrm{~h}$ showed limited cytoplasmic vacuoles (Fig. 2B-ii, upper panel) in contrast to the high level of vacu- 


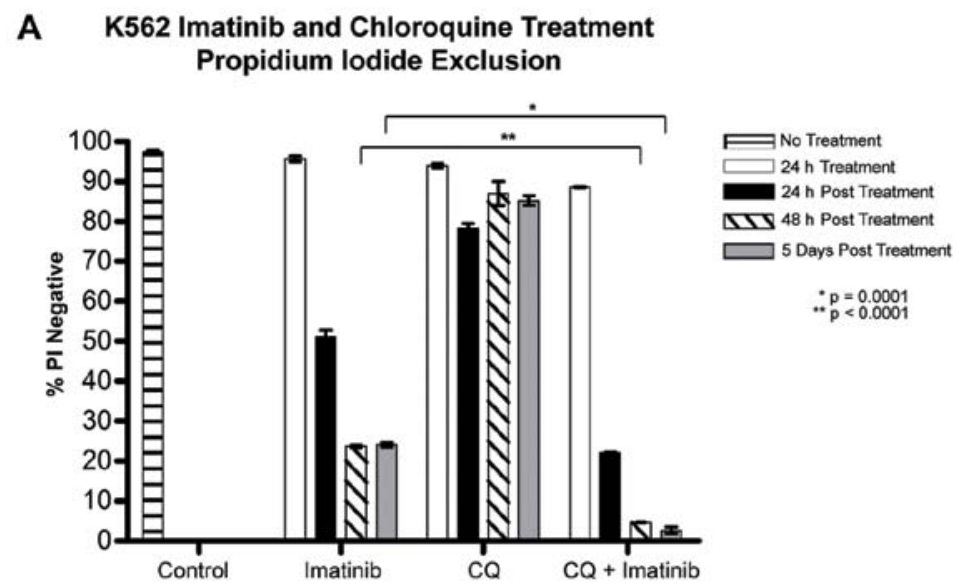

B i
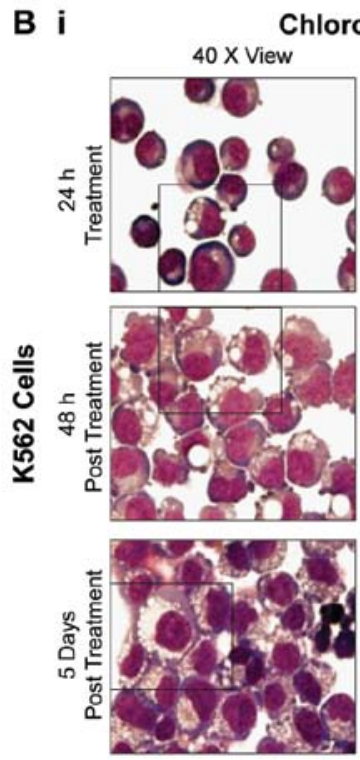

Chloroquine Detailed View
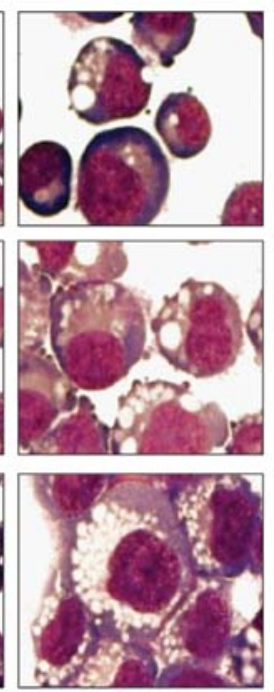

B ii
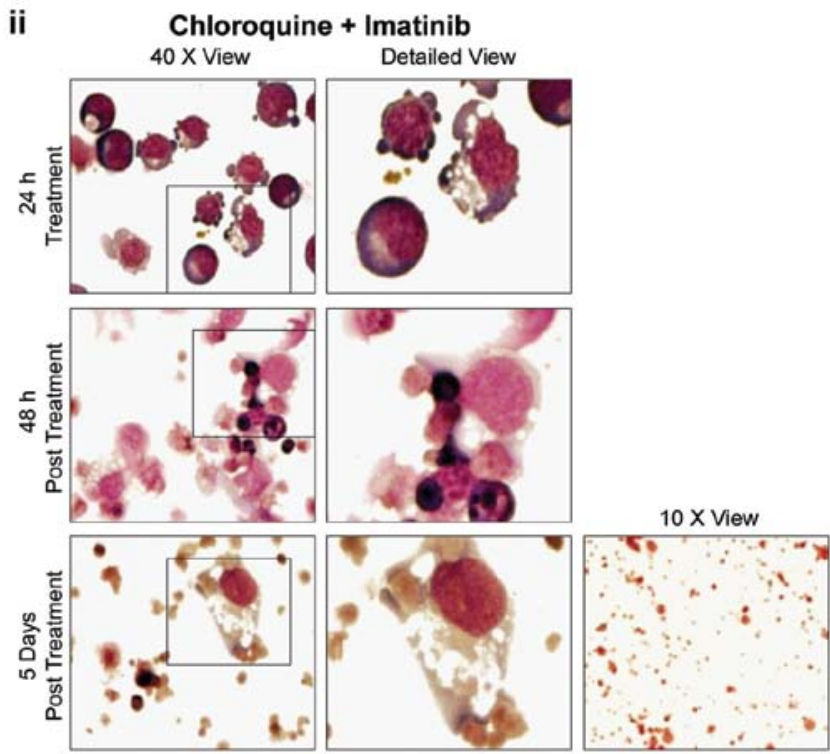

Figure 2. Effects of the combination of chloroquine and imatinib on cell viability and morphology. (A) Viability of K562 cells following treatment with $5 \mu \mathrm{M}$ imatinib, $25 \mu \mathrm{M}$ chloroquine or a combination of both treatments for $24 \mathrm{~h}$ and following drug withdrawal. The viability of cells treated with the combination was significantly reduced compared to the viability of cells treated with imatinib alone following drug withdrawal. (B) Morphology of K562 cells treated with (i) $25 \mu \mathrm{M}$ chloroquine or (ii) $5 \mu \mathrm{M}$ imatinib and $25 \mu \mathrm{M}$ chloroquine. Significant vesicular accumulation was noted in cells treated with chloroquine alone, or imatinib alone (Fig. 1A-ii), whereas this was significantly reduced in cells treated with the combination, and cells were fragmented or necrotic. Upper panels, 24-h treatment; middle panel, $48 \mathrm{~h}$ post treatment; lower panel, 5 days post treatment. Left hand column: magnification, $\mathrm{x} 40$; right hand column: enlarged section of left hand column indicated by a box. Third column of lower panel: magnification, x10 (5 days post treatment). CQ, chloroquine.

oles in cultures treated with imatinib alone (Fig. 1A-ii). At $48 \mathrm{~h}$ post imatinib and chloroquine combination treatment, cells exhibited a mixed morphology, with fragmented or intact nuclei, small dark shrunken cells and/or necrotic cells. At 5 days post treatment, only cell fragments remained (Fig. 2B-ii, middle and lower panel). The combination of chloroquine and imatinib was clearly a superior treatment when compared to either therapy alone, improving cytotoxicity and reducing the potential for cells to recover following treatment.

Vincristine and imatinib treatment. As vincristine limited the autophagy induced by imatinib (Fig. 1B and C), we investigated whether this agent improves cytotoxicity. Cells were treated for $24 \mathrm{~h}$ with $10 \mathrm{nM}$ vincristine, $5 \mu \mathrm{M}$ imatinib or a combination of both. Five days post drug removal, the viability of the cells treated with the combination of vincristine and imatinib was reduced to $3.5 \pm 0.1 \%$. This was statistically significant $(\mathrm{P}=0.0015)$ when compared to imatinib treatment alone (Fig. 3A)

Morphological analysis of vincristine-treated cells indicated that the majority of cells were arrested in mitosis at $24 \mathrm{~h}$ (Fig. 3B-i, left upper panel). In the cell treated with the combination of vincristine and imatinib, this morphology dominated and there was an absence of cytoplasmic vesicularisation (Fig. 3B-ii, right upper panel). At $48 \mathrm{~h}$ post vincristine treatment some cells exhibited tight clustering of cytoplasmic vacuoles (red arrows) and numerous multi-nucleated cells were evident (Fig. 3B-i, middle panel). This was also consistent with earlier LC3 staining in a limited number of cells, which appeared confined to a peri-nuclear area (Fig. 1B). The combination treatment at $48 \mathrm{~h}$ showed fewer cells with vesicles and many fragmented cells (Fig. 3B-ii, middle panel). Five days post treatment, cells treated with vincristine alone showed accumulation of cytoplasmic vesicles in the remaining intact cells, similar to cells 
A $\quad$ K562 imatinib and vincristine treatment propidium iodide exclusion

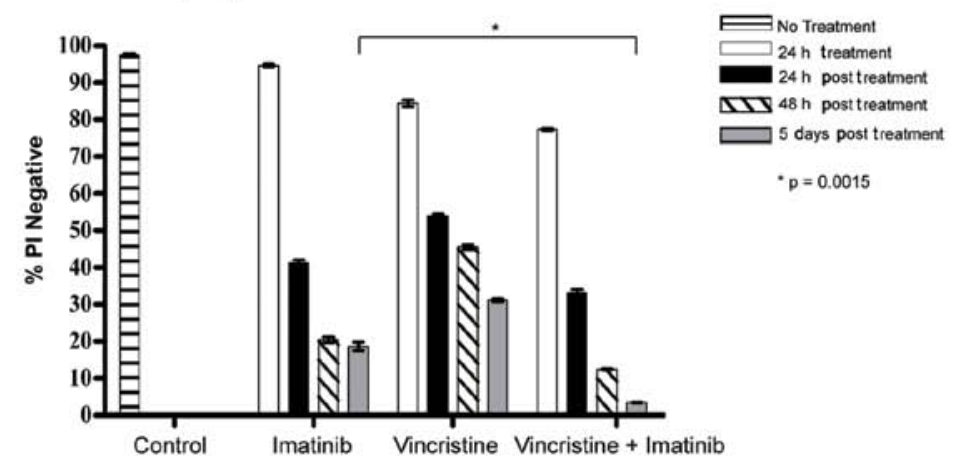

B i

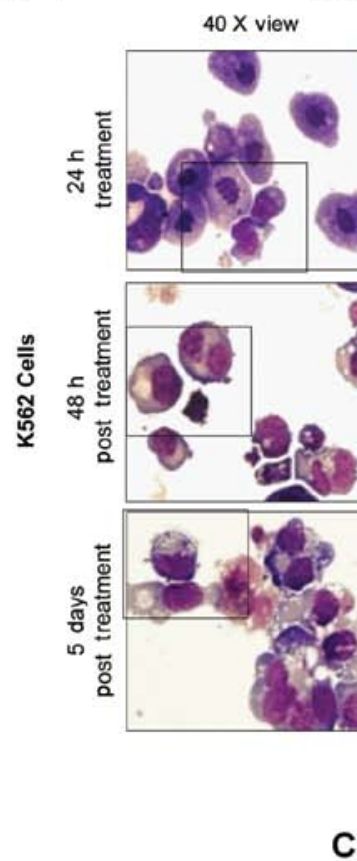

B ii

Detailed view
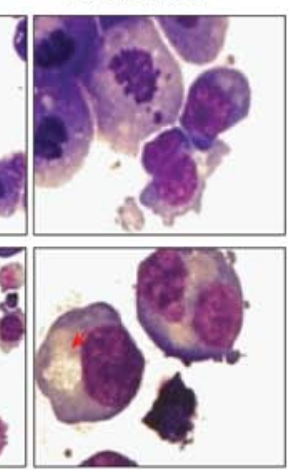

C

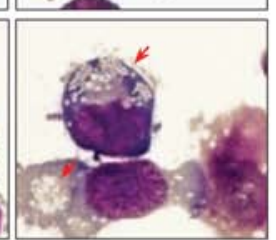

C
Vincristine + Imatinib $40 \times$ view Detailed view
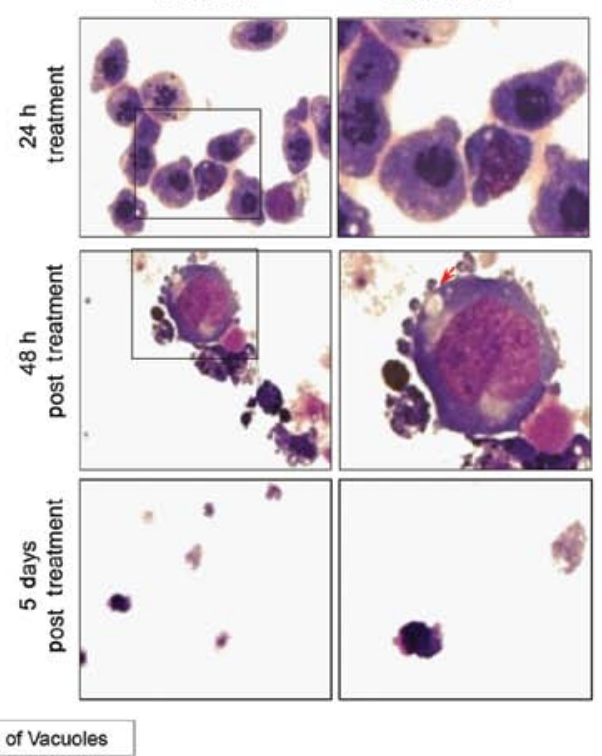

$K=$ Clustering of Vacuoles

Cell cycle analysis K562 cells $24 \mathrm{~h}$ treatment

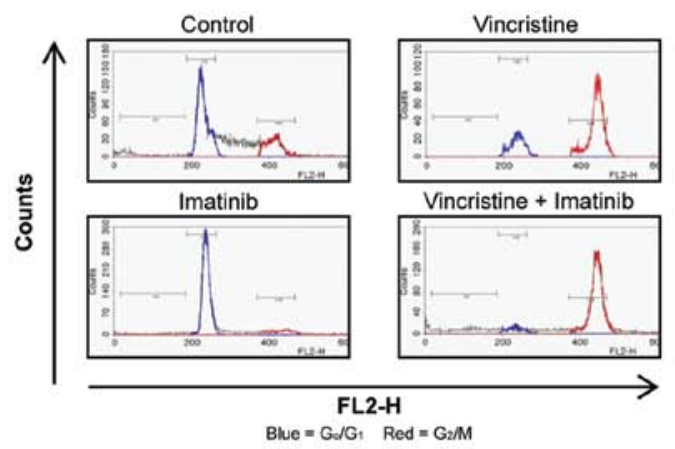

Figure 3. Effects of vincristine alone and in combination with imatinib on cell viability, morphology and cell cycle distribution. (A) Viability of K562 cells following treatment with $5 \mu \mathrm{M}$ imatinib, $10 \mathrm{nM}$ vincristine or a combination of both for $24 \mathrm{~h}$ and up to 5 days post drug withdrawal. The viability of the cells treated with the combination was significantly reduced compared to the viability of cells treated with imatinib alone following drug withdrawal. (B) Morphology of K562 cells following treatment with (i) $10 \mathrm{nM}$ vincristine, or (ii) combination of $5 \mu \mathrm{M}$ imatinib and $10 \mathrm{nM}$ vincristine. Cells treated with the combination treatment showed minor vesicle clustering in some cells and predominantly cell fragments at later time points. Upper panels, 24-h treatment; middle panel, $48 \mathrm{~h}$ post treatment; lower panel, 5 days post treatment. Left hand column: magnification, $\mathrm{x} 40$; right hand column: enlarged section of left hand columns indicated by a box. Red arrows, clustering of vacuoles. (C) Cell cycle analysis of K562 cells treated with imatinib, vincristine or both. All vincristine treated cells were arrested in $\mathrm{G}_{2} / \mathrm{M}$ phase. $\mathrm{x}$-axis, FL2-H; y-axis, cell number. Blue, $\mathrm{G}_{0} / \mathrm{G}_{1} ;$ red, $\mathrm{G}_{2} / \mathrm{M}$.

treated with imatinib, indicative of cells attempting recovery; some vesicular clustering was still evident (Fig. 3B-i, lower panel). The combination treatment showed only cell fragments and demonstrated a clear advantage over the single agents in reducing culture viability (Fig. 3B-ii, lower panel). Cell cycle analysis confirmed that a 24 -h treatment with vincristine with 


\section{A K562 Imatinib and Brefeldin A (BFA) Treatment} Propidium lodide Exclusion
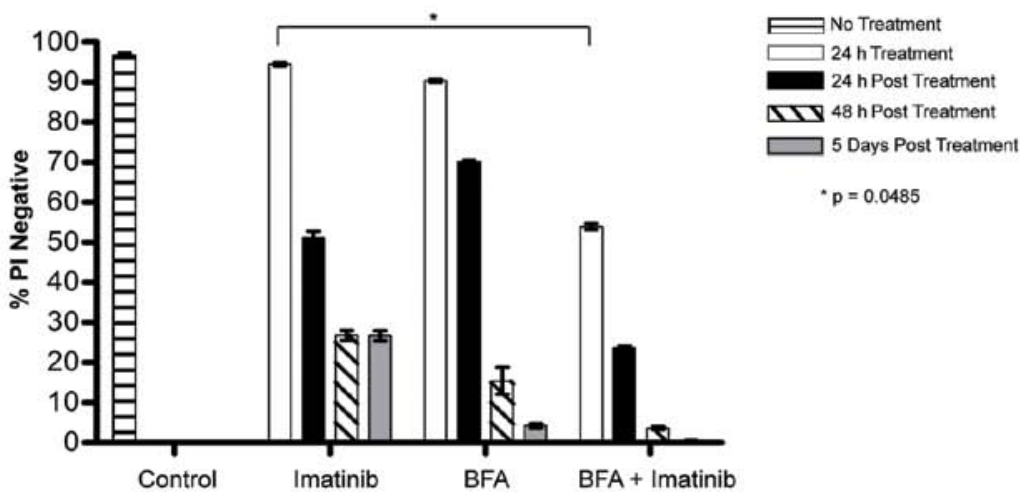

B i

Brefeldin A Brefeldin A + Imatinib

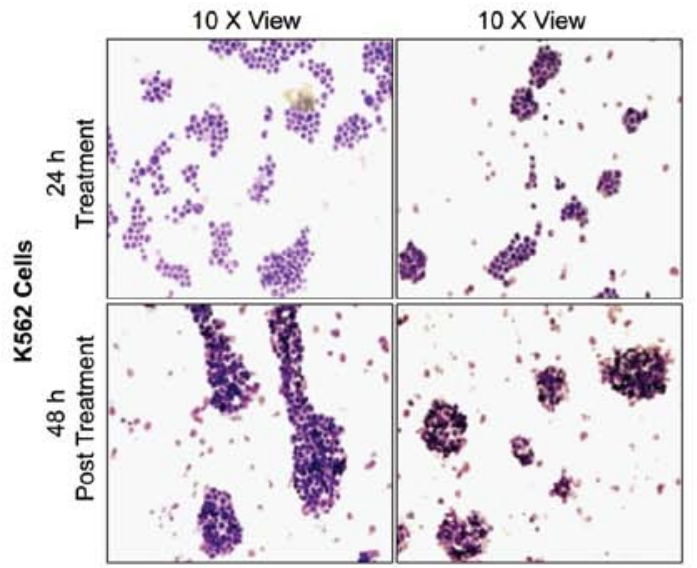

B ii

Brefeldin A

B iii

Brefeldin A + Imatinib
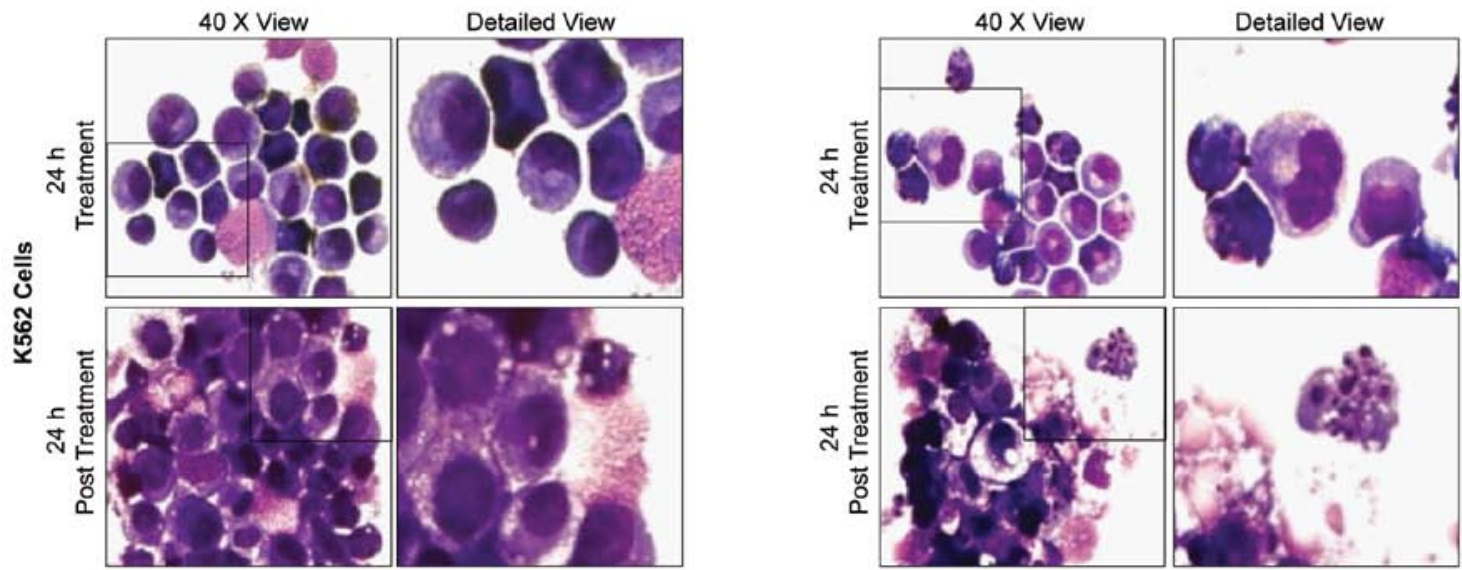

Figure 4. Effects of brefeldin A without and with imatinib on cell viability and morphology. (A) Viability of K562 cells following treatment with $5 \mu \mathrm{M}$ imatinib, $1 \mu \mathrm{g} / \mathrm{ml}$ brefeldin A or a combination of both for $24 \mathrm{~h}$ and following drug withdrawal. The viability of cells treated with the combination was reduced at 24 and $48 \mathrm{~h}$ post treatment compared to the viability of the cells treated with imatinib alone. At 5 days post treatment brefeldin A was cytotoxic when treated alone. (B-i) Morphology of K562 cells following treatment with $1 \mu \mathrm{g} / \mathrm{ml}$ brefeldin A or the combination of $5 \mu \mathrm{M}$ imatinib and $1 \mu \mathrm{g} / \mathrm{ml}$ brefeldin A. Clumping of the cells was evident (magnification, x10). Left column, BFA alone; right column, combination. Morphology of K562 cells at x40 following treatment with (ii) $1 \mu \mathrm{g} / \mathrm{ml}$ brefeldin A or (iii) combination of $5 \mu \mathrm{M}$ imatinib and $1 \mu \mathrm{g} / \mathrm{ml}$ brefeldin A. Cells treated with the combination showed evidence of necrosis following withdrawal of the drugs. Upper panels, 24-h treatment; lower panel, $24 \mathrm{~h}$ post treatment. Left hand column: magnification, $\mathrm{x} 40$; right hand column: enlarged section of left hand column indicated by a box. BFA, brefeldin A.

or without imatinib, arrested cells in the $\mathrm{G}_{2} / \mathrm{M}$ phase of the cell cycle (Fig. 3C).
Brefeldin A and imatinib treatment. Immunofluorescence of LC3 and Beclin 1 indicated that BFA inhibited autophagy 
(Fig. 1B and C). K562 cells were treated with $1 \mu \mathrm{g} / \mathrm{ml} \mathrm{BFA,}$ $5 \mu \mathrm{M}$ imatinib or a combination of both for $24 \mathrm{~h}$ and viability was assessed thereafter. When cells were treated with a combination of BFA and imatinib for $24 \mathrm{~h}$, viability decreased to $59.9 \pm 2.8 \%$ (Fig. $4 \mathrm{~A})(\mathrm{P}=0.0485$; combination treatment compared to imatinib treatment alone at $24 \mathrm{~h}$ ). At $48 \mathrm{~h}$ post drug withdrawal, viable cells were barely detectable following the combined BFA and imatinib treatment.

Analysis of cell morphology, following treatment with $\mathrm{BFA}$ as a single treatment or in combination with imatinib, showed K562 cells forming clusters (Fig. 4B-i). These clusters consisted of cells displaying a mixture of morphologies, the majority of which had intact (but condensed) nuclei and an absence of cytoplasmic vacuoles. A small number of cells $(<10 \%)$ exhibited nuclear fragmentation. At $24 \mathrm{~h}$ post treatment there was also evidence of necrosis (Fig. 4B-ii and -iii, lower panel). While the effect of BFA as a single agent was impressive, the response of cells to the combination therapy was more rapid.

\section{Discussion}

Following imatinib treatment, just under half of all patients achieve a complete molecular response (CMR) (15). However, after discontinuation of imatinib treatment, the majority of patients suffer relapse and recurrence of disease $(5,16)$. We and others have previously shown that the recovery of leukaemic cells is aided by autophagy and by inhibiting the autophagic response we can improve the cytotoxic impact of imatinib $(6,7)$. We, therefore, assessed whether disruption of other processes important for autophagy, reduces the recovery following imatinib withdrawal. The viability and recovery of CML-derived K562 cells were significantly reduced following the combination therapies.

The long-term viability of K562 cells treated with chloroquine and imatinib was significantly reduced when compared to long-term viability in cells treated with imatinib alone. Our analysis is consistent with another study of CML cell lines and CML patient samples which showed an improvement in imatinib-induced cytotoxicity with chloroquine (7). It is currently unclear, however, whether inhibition of late vesicular/lysosomal fusion is the sole reason for this enhancement. Our analysis of LC3 and Beclin 1 distribution in treated cells (at $24 \mathrm{~h}$ prior to cell death) showed reduced LC3 accumulation and substantially decreased Beclin 1 expression in the combination-treated cells. Reduced vesicular content was also evident. It is possible that this was a consequence of a lack of autophagosome turnover providing negative feedback to reduce autophagy initiation. Chloroquine derivatives have been prescribed for many years for other conditions such as malaria or for rheumatoid arthritis where hydroxylchloroquine is considered to be less toxic. Treatment regimens including hydroxyl-chloroquine have now entered CML clinical trials (10). It has also been incorporated in at least 12 clinical trials for solid tumours treated with various agents (reviewed in ref. 17).

The combination of imatinib and the microtubule inhibitor vincristine was more effective than either therapy alone and reduced the recovery of K562 cells following drug withdrawal. The arrested cells in the vincristine-treated populations also showed the greatest reduction in levels of both LC3 and Beclin 1. Mitotic inhibitors, which include vinca alkaloids as well as taxanes, are well established in the clinical setting and have formed the basis for a number of chemotherapeutic regimens. Vincristine has been incorporated in treatment regimens for acute leukaemias and blast crisis CMLs. It has also been combined with other non-targeted therapies such as cisplatin or etoposide for treatment of small-cell lung cancer (18). It is possible that these types of agents have dual activity: disruption of cell division and impairment of autophagy.

Treatment of K562 cells with BFA had the most dramatic effects on distribution of autophagy markers. Cytotoxicity was accelerated in cells treated with a combination of BFA and imatinib; however, BFA was also highly effective alone 5 days after drug withdrawal. BFA has been reported to be effective against pancreatic, gliobastoma and prostate cancer (19-21). A generation of new derivatives such as breflate (22-24) is overcoming problems associated with the low water solubility of BFA, making it an interesting candidate for future clinical trials.

We previously showed that specific autophagy knockdown (siRNA approach) reduced the recovery of imatinib-treated cells (6). However, in the combinations presented here we cannot conclusively attribute the enhanced cytotoxicity of the combined treatments with imatinib to autophagy inhibition. These agents have other activities. The aim of the present study was to indicate that certain pharmacological agents can also impede autophagy as part of their activity and this may be an important factor in the design of combination treatment regimes where the efficacy of one of the agents is limited by autophagy.

Newer TKIs such as nilotinib (Bcr-Abl inhibitor) or dasatinib and INNO-406 (src family kinase inhibitors) developed to improve treatment of imatinib-intolerant or -resistant leukaemia have also been reported to be limited by autophagy $(7,25)$. We and others have also shown that the induction of autophagy is not limited to the treatment with TKIs but also with other nontargeted therapies such as VP16 or SAHA $(6,26)$. Autophagy has also been reported to be a barrier in achieving successful treatment of other cancer types, including breast, prostate and oesophageal cancer treated with tamoxifen, sorafenib or 5-fluorouracil (27-29).

An important part of the rationale for using autophagy inhibitors in cancer is the potential for the eradication of the more resistant residual cells and importantly the transformed stem cells which can lead to re-population of disease. Hydroxylchloroquine has been reported to be effective at targeting primitive CML cells including colony forming cells and longterm culture-initiating cells (7). Notably, autophagy has been reported to be important for normal stem cell longevity (30). This suggests that normal cells may also be affected by prolonged treatment with autophagy inhibitors. These studies highlight the need for the development of more selective inhibitors and a better understanding of their effects on normal cells for effective clinical management.

The present study suggests that the early implementation of regimes which incorporate an autophagy-deregulating agent in combination with TKIs may reduce the residual disease present in CML. Importantly, this will remain largely targeted if the autophagy inhibitor has limited toxicity on its 
own. Regimes designed to reduce autophagy may limit the development of TKI resistance and the toxicity associated with dose escalation or less targeted treatments.

\section{Acknowledgements}

We are eternally grateful to the late Professor Gerald C. O'Sullivan, Cork Cancer Research Centre, for helpful discussions and support throughout this study. We also thank Dr Baukje Elzinga, Cork Cancer Research Centre, for her input and advice. This study was funded by the Children's Leukaemia Research Project, Cancer Research Ireland, the Higher Education Authority of Ireland and the Cork Cancer Research Centre.

\section{References}

1. Nowell PC and Hungerford DA: A minute chromosome in human chronic granulocyte leukeamia. Science 132: 1497-1501, 1960.

2. Melo JV: The diversity of BCR-ABL fusion proteins and their relationship to leukemia phenotype. Blood 88: 2375-2384, 1996.

3. Calabretta B and Perrotti D: The biology of CML blast crisis. Blood 103: 4010-4022, 2004.

4. Quentmeier H, Eberth S, Romani J, Zaborski M and Drexler HG BCR-ABL1-independent PI3Kinase activation causing imatinibresistance. J Hematol Oncol 4: 6, 2011.

5. Cortes J, O'Brien S and Kantarjian H: Discontinuation of imatinib therapy after achieving a molecular response. Blood 104: 2204-2205, 2004.

6. Crowley LC, Elzinga BM, O'Sullivan GC and McKenna SL: Autophagy induction by Bcr-Abl-expressing cells facilitates their recovery from a targeted or nontargeted treatment. Am J Hematol 86: 38-47, 2011.

7. Bellodi C,Lidonnici MR, Hamilton A, et al: Targeting autophagy potentiates tyrosine kinase inhibitor-induced cell death in Philadelphia chromosome-positive cells, including primary CML stem cells. J Clin Invest 119: 1109-1123, 2009.

8. Mizushima N: Autophagy: process and function. Genes Dev 21: 2861-2873, 2007.

9. Degtyarev M,De Maziere A, Orr C, et al: Akt inhibition promotes autophagy and sensitizes PTEN-null tumors to lysosomotropic agents. J Cell Biol 183: 101-116, 2008.

10. Holyoakes T: Medical Research Council Research Portofolio: A randomised phase II trial of IM versus HCQ/IM for patients with CML in MCyR with residual disease by Q-PCR. http://www. mrc.ac.uk/ResearchPortfolio/Grant/Record.htm?GrantRef=G09 $00882 \&$ CaseId $=15301$.

11. Eskelinen EL, Prescott AR, Cooper J, et al: Inhibition of autophagy in mitotic animal cells. Traffic 3: 878-893, 2002.

12. Groth-Pedersen L, Ostenfeld MS, Hoyer-Hansen M, Nylandsted J and Jaattela M: Vincristine induces dramatic lysosomal changes and sensitizes cancer cells to lysosome-destabilizing siramesine. Cancer Res 67: 2217-2225, 2007.

13. Vetterlein M, Niapir M, Ellinger A, Neumüller J and Pavelka M: Brefeldin A-regulated retrograde transport into the endoplasmic reticulum of internalised wheat germ agglutinin. Histochem Cell Biol 120: 121-128, 2003.

14. Nishida Y, Arakawa S, Fujitani K, et al: Discovery of Atg5/Atg7independent alternative macroautophagy. Nature 461: 654-658, 2009.
15. Aguayo A and Couban S: State-of-the-art in the management of chronic myelogenous leukemia in the era of the tyrosine kinase inhibitors: evolutionary trends in diagnosis, monitoring and treatment. Leuk Lymphoma 50 (Suppl 2): 1-8, 2009.

16. O'Brien SG, Guilhot F, Goldman JM, et al: International randomized study of interferon versus STI571 (IRIS) 7-year follow-up: sustained survival, low rate of transformation and increased rate of major molecular response (MMR) in patients (pts) with newly diagnosed chronic myeloid leukemia in chronic phase (CMLCP) treated with imatinib (IM). (ASH Annual Meeting Abstracts). Blood 112: 186, 2008.

17. Amaravadi RK, Lippincott-Schwartz J, Yin X-M, et al: Principles and current strategies for targeting autophagy for cancer treatment. Clin Cancer Res 17: 654-666, 2011.

18. Lassen U, Kristjansen PE, Osterlind K, et al: Superiority of cisplatin or carboplatin in combination with teniposide and vincristine in the induction chemotherapy of small-cell lung cancer. A randomized trial with 5 years follow up. Ann Oncol 7: 365-371, 1996.

19. Pommepuy I, Terro F, Petit B, et al: Brefeldin A induces apoptosis and cell cycle blockade in glioblastoma cell lines. Oncology 64: 459-467, 2003.

20. Chapman JR, Tazaki H, Mallouh C and Konno S: Brefeldin A-induced apoptosis in prostatic cancer DU-145 cells: a possible p53-independent death pathway. BJU Int 83: 703-708, 1999.

21. Larsson DE, Wickström M, Hassan S, OBerg $K$ and Granberg DAN: The cytotoxic agents NSC-95397, brefeldin A, bortezomib and sanguinarine induce apoptosis in neuroendocrine tumors in vitro. Anticancer Res 30: 149-156, 2010.

22. Fox BM, Vroman JA, Fanwick PE and Cushman M: Preparation and evaluation of sulfide derivatives of the antibiotic brefeldin A as potential prodrug candidates with enhanced aqueous solubilities. J Med Chem 44: 3915-3924, 2001.

23. Phillips LR, Wolfe TL, Malspeis L and Supko JG: Analysis of brefeldin $\mathrm{A}$ and the prodrug breflate in plasma by gas chromatography with mass selective detection. J Pharm Biomed Anal 16: 1301-1309, 1998.

24. Anadu NO, Davisson VJ and Cushman M: Synthesis and anticancer activity of brefeldin A ester derivatives. J Med Chem 49: 3897-3905, 2006.

25. Kamitsuji Y, Kuroda J, Kimura S, et al: The Bcr-Abl kinase inhibitor INNO-406 induces autophagy and different modes of cell death execution in Bcr-Abl-positive leukemias. Cell Death Differ 15: 1712-1722, 2008

26. Carew JS, Nawrocki ST, Kahue CN, et al: Targeting autophagy augments the anticancer activity of the histone deacetylase inhibitor SAHA to overcome Bcr-Abl-mediated drug resistance. Blood 110: 313-322, 2007.

27. Qadir MA, Kwok B, Dragowska WH, et al: Macroautophagy inhibition sensitizes tamoxifen-resistant breast cancer cells and enhances mitochondrial depolarization. Breast Cancer Res Treat 112: 389-403, 2008.

28. Ullen A, Farnebo M, Thyrell L, et al: Sorafenib induces apoptosis and autophagy in prostate cancer cells in vitro. Int J Oncol 37: $15-20,2010$.

29. O'Donovan TR, O'Sullivan GC and McKenna SL: Induction of autophagy by drug-resistant esophageal cancer cells promotes their survival and recovery following treatment with chemotherapeutics. Autophagy 7: 509-524, 2011

30. Mortensen M, Soilleux EJ, Djordjevic G, et al: The autophagy protein Atg7 is essential for hematopoietic stem cell maintenance. J Exp Med 208: 455-467, 2011. 